\title{
Fetal demise of the remaining co-twin of monoamniotic twins due to cord entanglement after the death of the first twin
}

\begin{abstract}
Umbilical cord entanglement is the leading cause of fetal mortality in monoamniotic twin pregnancies. Published methods for detecting this complication include color Doppler (single and dual gate), pulsed Doppler, and three dimensional sonography)3D $\mathrm{U} / \mathrm{S}$ ) however; no method provides diagnosis with certainty. In this case, we report 25-year-old G2 P1 was diagnosed as monoamniotic monochorionic twin pregnancy at 12 weeks gestation. At 16 weeks one of the co-twins died. At 35th week gestation, an unexpected fetal death of the remaining co-twin was diagnosed. The umbilical cord of the dead co-twin was found to be constricted by the macerated umbilical cord.

\section{Highlights}

a. Monoamniotic twins are associated with high fetal mortality mainly due to cord entanglement.

b. In monoamniotic twins the early fetal loss of one of co-twins may give false impression that the remaining co-twin is not in danger of cord entanglement.

c. This report highlights the spots on the importance of cord transection in monoamniotic twins after the death of one of co-twins.
\end{abstract}

Keywords: monoamniotic twins, cord entanglement, fetal monitoring, fetal death
Volume 5 Issue 3 - 2019

\author{
Adel E Elgergawy,' Mona khalid,' Manal Abd \\ Raof $^{2}$ \\ 'Assistant professor of Obstetrics and Gynecology, Tanta \\ University, Egypt \\ ${ }^{2}$ Lecturer of Obstetrics and Gynecology, Tanta University, Egypt \\ Correspondence: Adel Elshhat Elgergawy, Department \\ of Obstetrics and Gynecology, Faculty of Medicine, Tanta \\ University, Tanta, Postal code: 3 I I I I, Egypt, Tel +20 I002057250, \\ Email dradelali775@gmail.com
}

Received: May II, 2019 | Published: June 05, 2019

\section{Introduction}

The prevalence of monoamniotic twins ranges between 1 in 5000 and 1 in 25,000 pregnancies. $1 \%$ of monozygotic twins are monoamniotic, occurring when 2 fetuses coexist inside one amnion and the cleavage between 8 and 13 days after fertilization ${ }^{1}$ prematurity, umbilical cord entanglement, twin to twin transfusion syndrome, congenital anomalies, and intrauterine growth restriction are the leading causes of high $(8-42 \%)$ peri-natal mortality of monoamniotic pregnancies. Nearly in $100 \%$ of these cases umbilical cord entanglement was diagnosed. ${ }^{2}$ umbilical cord strangulation may leads to more than $50 \%$ of fetal death rate, its precise evaluation and treatment are very essential to enhance favorable outcomes. ${ }^{3}$ because of dividing membranes in monochorionic diamniotic pregnancies, cord strangulation which is a leading cause of fetal mortality in monoamniotic pregnancies is not a complication. ${ }^{4}$ In spite of this complication can be diagnosed by a variety of methods include color Doppler, 3D U/S and pulsed Doppler sonography; no method provides diagnosis with high certainty, making the diagnosis uncertain in some cases. ${ }^{2}$ In this case, we report unexpected fetal demise of the normal co-twin at 35 weeks gestation, after the death of the first co-twin and now we report it with short review.

\section{Case report}

Pregnant woman 25-year-old, G2 P1, previous caesarean section (C.S) attended to our department at 12 weeks gestation after normal spontaneous pregnancy. Abdominal Voluson E8 2D ultrasound was done showing that the twins were within the average gestational age at that time. Twins were monochorionic-monoamniotic, and were females. At this visit both umbilical cords were visibly separated from each other. Due to high risk of fetal demise resulting from umbilical cord entanglement we did counseling for parents. Then during weekly visits at the outpatient clinic death of one co-twin was noticed at 16 weeks gestation, the living co-twin was completely normal with no anomalies in the ordinary weekly scanning, especially neurological examination. Regular antenatal fetal assessment including non-stress test, Doppler study of the umbilical cord and amniotic fluid index starting from 28 weeks, and repeated every week which were normal. A 30 weeks of pregnancy the patient refused hospitalization which was recommended for intensive observation. A 35 week gestation during routine outpatient visits, unexpected death of co-twin was detected, after patient counseling and performance of complete laboratory investigations, caesarean section was performed according to the patient request. After the delivery of the fetus, its cord was found to be tightly constricted by the cord of the macerated fetus (fetus papyraceous). By gross examination of the placenta, insertion of the both cords is central and were very close to each other. As shown in the following Figure 1.

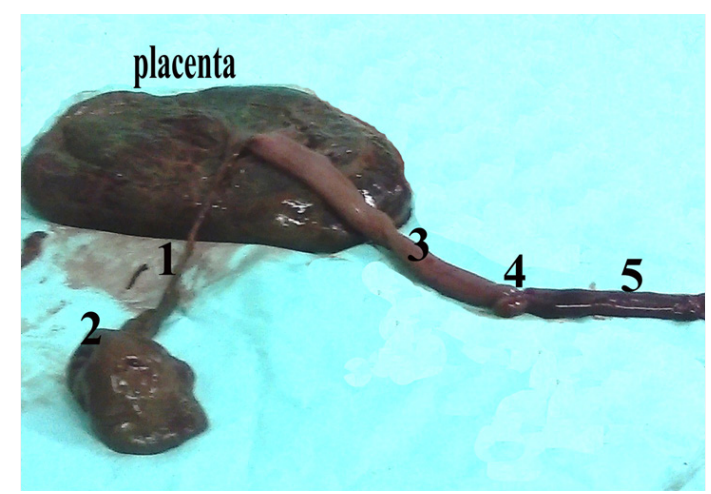

Figure I I-cord of macerated fetus, 2-macerated fetus( papyraceous), 3-pale (maternal side) part of the cord of the cord of the second dead twin, 4-site of constriction (entanglement), 5-congested part of the cord of the second twin. 


\section{Discussion}

We report a case of monoamniotic diagnosed early in the first trimester in which, death of the first co-twin in the early second trimester resulting in the death of the remaining of co-twin in the late third trimester as a result of cord entanglement. False sense from death of one of two co-twins is the real cause of the death of the remaining co-twins. During the first trimester of pregnancy, accurate diagnosis of Monoamniotic twins made if 2 embryos or fetuses with one yolk sac are found on the ultrasound image. After that they can be diagnosed, if there is no dividing amniontic memebrane between twins with a single placenta and both are of the same gender. ${ }^{3}$ In more than two thirds of monoamniotic twins, Cord strangulation is present, mostly in the first trimester, as there is excess amnion in relation to the fetal body masses. ${ }^{5}$ Amniocentesis, or administration of sulindac have been described to lower perinatal mortality from cord entanglement. ${ }^{6}$ In monoamniotic pregnancies, during the course of pregnancy 2 fetuses are freely mobile in one amniotic sac so cord strangulation is inevitable complication. It is too difficult to consider that cord strangulation was the only cause of fetal death as it is present nearly in all cases. ${ }^{6}$ As fetal viability is reached at 26 weeks of gestation in monoamniotic twins We can start intensive antenatal fetal monitoring with the aim of the prevention of fetal death. Diagnosis of cord strangulation in monoamniotic twins should be as early (prenatally) as possible, and when it is considered, it is mandatory to explore the cord for signs of compression or occlusion should be done.

Although Sherer denoted that cord strangulation can be diagnosed using the transvaginal 2D color Doppler $\mathrm{U} / \mathrm{S}$ early in the first trimester, it was difficult to confirm umbilical cord entanglement with 2D ultrasound. ${ }^{7}$ But dual-gate Doppler is easier and more accurate in umbilical cord entanglement diagnosis. (4) The 3D U/S provide good visualization of the cord strangulation and help to explain the magnitude of the problem and share the parents in decision making. As most 3D U/S is performed late in pregnancy usually after 28 weeks gestation, so the cases of cord entanglement that can be diagnosed using 3D U/S have been rarely reported. ${ }^{8}$ Yun Sung Jo et al., ${ }^{9}$ used 3D U/S in the first trimester to diagnose first case with umbilical cord entanglement.

In case of discordant fetal anomaly, as a result of acute perimortem blood loss into the dying co-twin of a monochorionic twin, demise of the normal co-twin is an actual threat. So selective feticide should be considered but sudden fetal demise of normal co-twin from cord entanglement remains a risk. ${ }^{10}$ Cord occlusion which has been widely considered selective feticide policy for treatment of diamniotic monochorionic twin pregnancies in case of twin with reversed arterial perfusion (TRAP),discordant congenital anomaly, or a critically ill fetus with severe TTTS, ${ }^{11}$ Cord Transection has been postulated as an additional treatment option in monoamniotic and pseudo monoamniotic twins, to minimize the risks of cord entanglement later in pregnancy. ${ }^{12} \mathrm{So}$, it is necessary to perform transection of the occluded cord to save remaining normal co-twin. ${ }^{13}$

It was found that from 11 weeks of gestation prospective risk of IUFD decreased greatly between 28 and 30 weeks of gestation in monoamniotic twins. Then at 32-34 weeks gestation this risk is markedly increased, so, this risk to fetuses exceeding the risk of postnatal complications at 32 weeks gestation. So, it is considered to advise fetal delivery at 32 weeks gestation, ${ }^{14}$ and daily antenatal inpatient fetal surveillance with elective delivery at 32 weeks has been suggested. ${ }^{15}$ From this case, management should consist of intensified fetal monitoring (with hospitalization at 26 weeks) with daily fetal cardiotocography (CTG), Increased attention be paid to caliber alterations or flow abnormalities in the umbilical cord of the remaining co-twin during further weekly follow-up Doppler U/S examinations and caesarean section at 32 weeks gestation. We therefore recommend that echogenic remains of the umbilical cord of the demised fetus be looked at, as the umbilical cord of the demised fetus will not show Doppler flows anymore. In conclusion, this case demonstrates that in monochorionic twins, risk for cord entanglement leading to a fatal outcome for the remaining co-twin is a real threat even after the death of the first co-twin early in pregnancy and transection of the cord provide the golden solution for this highly fetal complication if available.

\section{Consent}

Written consent taken from patient for publication

\section{Acknowledgments}

None.

\section{Conflicts of interest}

All authors declare no conflict of interest.

\section{References}

1. Beasly E, Megerian G, Gerson A, et al. Monoamniotic twins. case series and proposal for antenatal management. Obstet Gynecol. 1999;93(1):130 134.

2. Dias T, Mahsud-Dornan S, Bhide A, et al. Cord entanglement and perinatal outcome in monoamniotic twin pregnancies. Ultrasound Obstet Gynecol. 2010;35:201-204

3. Pasquini L, Wimalasundera RC, Fisk NM. Management of other complications specific to monochorionic twin pregnancies. Best Pract Res Clin Obstet Gynaecol. 2004;18:577-599.

4. Ayumu Ito, Masahiko Nakata, Ayako Oji, et al. Diagnosis of umbilical cord entanglement in a monochorionicdiamniotic twin pregnancy with spontaneous septostomyof the dividing membranes using dual-gate Doppler imaging. J Med Ultrasonics. 2018;45(1):89-192.

5. Sebire NJ, Souka A, Skentou H, et al. First trimester diagnosis of monoamniotic twin pregnancies. Ultrasound Obstet Gynecol. 2000;16:223-225

6. Pasquini L, Wimalasundera RC, Fichera A, et al. High perinatal survival in monoamniotic twins managed by prophylactic sulindac, intensive ultrasound surveillance, and Cesarean delivery at 32 weeks' gestation. Ultrasound Obstet Gynecol. 2006;28:681-687.

7. Sherer DM, Sokolovski M, Haratz-Rubinstein N. Diagnosis of umbilical cord entanglement of monoamniotic twins by first-trimester color doppler imaging. J Ultrasound Med. 2002;21:1307-1309.

8. Kuwata T, Matsubara S, Suzuki M. 3D color Doppler of mon- oamniotic twin cord entanglement. Arch Gynecol Obstet. 2010;281:973-974.

9. Yun Sung Jo, Hyun Joo Son, Dong Gyu Jang, et al. Monoamniotic twins with one fetal anencephaly and cord entanglement diagnosed with three dimentional ultrasound at 14 weeks gestation. Int J Med Sci. 2011;8(7):573-576.

10. Fusi L, McParland P, Fisk N, et al. Acute twin-twin transfusion a possible mechanism for brain-damaged survivors after intrauterine death of a monochorionic twin. Obstet Gynecol. 1981;78:517-520. 
11. Ville Y.Selective feticide in monochorionic pregnancies: toys for the boys or standard care? Ultrasound Obstet Gynecol. 1991;22:448-450.

12. Vandenbussche FPHA, Deprest JA, Klumper FJCM, et al. Minimallyinvasive intrauterine treatment in four monochorionic twin pregnancies complicated by an acardiac. Ned Tijdschr Geneesk. 2003;147:931-936

13. Quintero R, Morales W. Percutaneous fetal intervention. In: Harrison M editor. The Unborn Patient. Philadelphia, Saunders. 1999;3:100-105.
14. Van Mighem T, De Heus R, Lewi L, et al. Prenatal management of monoamniotic twin pregnancies. Obstet Gynecol. 1998;179:788-793.

15. Ezra Y, Shveiky D, Ophir E, et al. Intensive management and early delivery reduce antenatal mortality in monoamniotic twin pregnancies. Acta Obstet Gynecol Scand. 2005;84:432-435. 